\title{
Levels of soluble receptor for AGE are cross-sectionally associated with cardiovascular disease in type 1 diabetes, and this association is partially mediated by endothelial and renal dysfunction and by low-grade inflammation: the EURODIAB Prospective Complications Study
}

\author{
J. W. M. Nin • I. Ferreira • C. G. Schalkwijk • \\ M. H. Prins • N. Chaturvedi • J. H. Fuller • \\ C. D. A. Stehouwer • \\ EURODIAB Prospective Complications Study Group
}

Received: 16 September 2008 / Accepted: 29 December 2008 / Published online: 30 January 2009

(C) The Author(s) 2009. This article is published with open access at Springerlink.com

\begin{abstract}
Aims/hypothesis Plasma soluble receptor for AGE (sRAGE) may reflect the activity of the AGE-RAGE axis, which has been proposed as a potential mechanism linking hyperglycaemia to vascular complications in diabetes. We have therefore investigated: (1) whether sRAGE is associated with greater prevalence of cardiovascular disease (CVD) and microvascular complications in type 1 diabetic individuals; and (2) the extent to which any such associations are explained by markers of endothelial and renal dysfunction and inflammation.
\end{abstract}

Electronic supplementary material The online version of this article (doi:10.1007/s00125-009-1263-5) contains supplementary material, which is available to authorised users.

J. W. M. Nin · I. Ferreira • M. H. Prins

Care and Public Health Research Institute (CAPHRI),

Maastricht University Medical Centre,

Maastricht, the Netherlands

J. W. M. Nin • I. Ferreira • C. G. Schalkwijk • C. D. A. Stehouwer Cardiovascular Research Institute Maastricht (CARIM),

Maastricht University Medical Centre,

Maastricht, the Netherlands

J. W. M. Nin $(\bowtie) \cdot$ I. Ferreira $\cdot$ C. G. Schalkwijk •

C. D. A. Stehouwer $(\bowtie)$

Department of Internal Medicine,

Maastricht University Medical Centre,

P. Debyelaan 25, P.O. Box 5800, 6202 AZ, Maastricht,

The Netherlands

e-mail: j.nin@intmed.unimaas.nl

e-mail: cda.stehouwer@mumc.nl
Methods The study included 477 individuals (234 women; mean age $42 \pm 10$ [SD] years) from the EURODIAB Prospective Complications Study. We used linear regression analyses to investigate the differences in SRAGE levels between individuals with and without vascular complications. All analyses were adjusted for age, sex, $\mathrm{HbA}_{1 \mathrm{c}}$, duration of diabetes and other risk factors.

Results Individuals with CVD $(n=116)$ had higher levels of sRAGE than those without CVD or any microvascular complications $(n=178): \beta=0.15 \quad(95 \%$ CI $0.04-0.27)$.

I. Ferreira $\cdot$ M. H. Prins

Department of Clinical Epidemiology and

Medical Technology Assessment,

Maastricht University Medical Centre,

Maastricht, the Netherlands

N. Chaturvedi

International Centre for Circulatory Health,

National Heart and Lung Institute,

Imperial College London,

London, UK

J. H. Fuller

Department of Epidemiology and Public Health,

Royal Free and University College London Medical School,

London, UK 
Further adjustments for markers of endothelial $(\beta=0.13$ $[0.02-0.24])$ and renal dysfunction $(\beta=0.10[-0.01,0.20])$ and inflammation $(\beta=0.12[0.01-0.23])$ attenuated these differences; altogether these variables explained about $50 \%$ of the association between sRAGE and prevalent CVD. sRAGE levels tended to be higher in the presence and across the levels of severity of albuminuria ( $p$ for trend= 0.087 ) and retinopathy ( $p$ for trend $=0.057$ ); adjustments for endothelial and renal dysfunction and inflammation also attenuated these differences.

Conclusions/interpretation sRAGE is associated with greater prevalence of CVD in type 1 diabetic individuals, and these associations may be partly explained by endothelial and renal dysfunction and low-grade inflammation.

Keywords Cardiovascular disease - Diabetes mellitus · Endothelial dysfunction · Inflammation · Microvascular complications $\cdot$ Renal dysfunction $\cdot$ Soluble receptor for advanced glycation end-products

\begin{tabular}{ll}
\multicolumn{2}{l}{ Abbreviations } \\
CRP & C-reactive protein \\
CVD & Cardiovascular disease \\
eGFR & Estimated GFR \\
esRAGE & Endogenous secretory receptor for AGEs \\
IMT & Intima-media thickness \\
RAGE & Receptor for AGEs \\
sE-selectin & Soluble E-selectin \\
sRAGE & Soluble receptor for AGEs \\
sVCAM-1 & Soluble vascular cell adhesion molecule-1 \\
UAE & Urinary albumin excretion rate
\end{tabular}

\section{Introduction}

The increased cardiovascular disease (CVD) risk in individuals with diabetes mellitus cannot be fully explained by traditional cardiovascular risk factors $[1,2]$. Therefore, other factors and mechanisms potentially involved need to be explored. At the molecular level, one pathway through which hyperglycaemia may lead to vascular complications is the formation of AGEs. An important mechanism through which AGEs induce vascular disease is by binding to their specific receptors [3].

Several different receptors for AGEs have been identified, one of which is termed RAGE. Different cell types including human endothelial cells express RAGE [4]. In addition to cell-bound RAGE, soluble forms of RAGE appear in the plasma $[4,5]$, as different splice variants of RAGE (e.g. endogenous secretory receptor for AGE [esRAGE]) leaking through the transmembrane and cytosolic domain $[4,6]$, and as a proteolytically cleaved form of
RAGE (sRAGE), which is most probably shed into the circulation by the sheddase, known as a disintegrin and metalloprotease 10 (ADAM 10) [7]. The functional role of these soluble forms of RAGE in the circulation remains unclear, but they may reflect the activity of the AGERAGE axis. The ligation of RAGE activates the endothelial cell and triggers multiple signalling cascades [8, 9], resulting in activation and translocation of nuclear transcription factors and transcription of the target genes, including adhesion molecules [9, 10] and proinflammatory cytokines [11]. The activation of RAGE may also lead to nephropathy [12]. We have therefore hypothesised that the activation of the AGE-RAGE axis may lead to vascular complications in diabetes through increases in endothelial and renal dysfunction and low-grade inflammation.

Evidence so far with regard to the association between soluble forms of RAGE and vascular disease in diabetes is contradictory, with several studies showing positive [1317], but also inverse associations [18, 19]. The fact that these studies have investigated either esRAGE $[18,19]$ or sRAGE [13-17] may explain the discrepancies. The few studies that have examined both soluble forms of RAGE have shown that sRAGE, but not esRAGE, is positively associated with albuminuria in individuals with type 2 diabetes [16], whereas esRAGE and sRAGE were inversely associated with intima-media thickness (IMT), a surrogate marker of atherosclerosis [20] and its progression [21] in individuals with type 1 diabetes. In addition, the extent to which mechanisms such as endothelial and renal dysfunction and low-grade inflammation, which are intertwined and related to macro- and microvascular complications in diabetes [22-24], underlie the association between sRAGE and vascular complications (if any) has never been investigated.

In view of these considerations, we have investigated, first, whether sRAGE levels are associated with a greater prevalence of macro- and microvascular complications in individuals with type 1 diabetes; and second, the extent to which any such associations are explained (i.e. are mediated) by markers of endothelial and renal dysfunction and low-grade inflammation.

\section{Methods}

Participants and study design

We used data from the EURODIAB Prospective Complications Study, which is a follow-up of the EURODIAB IDDM Complications Study that has been described in detail elsewhere [25]. Briefly, baseline investigations were performed between 1989 and 1991 on 3,250 individuals with type 1 diabetes, defined as a clinical diagnosis made before the age of 36 years, and needing continuous insulin therapy 
within 1 year of diagnosis. The individuals, aged between 15 and 60 years, were recruited from 31 centres in 16 European countries. Sample selection was stratified by sex, age group and duration of diabetes, to ensure sufficient representation in all categories. All individuals gave informed consent and the study was approved by the local Ethics Committees [25]. These individuals were invited for a follow-up examination on average 7-9 years after the baseline examinations. Of the 3,250 included individuals, 1,880 (57.8\%) returned for reexamination [26-28]. At follow-up, a cross-sectional nested case-control study of markers of inflammation and endothelial dysfunction and their associations with complications was performed in a subset of individuals $(n=543)[22,23$, 29-33]. Cases were selected as those with a greatest vascular complication burden as possible $(n=348)$ and controls were selected as those who were completely free of any complications (i.e. with no evidence of CVD, albuminuria and retinopathy) $(n=195)$. This selection allowed us to compare individuals with single or multiple complications with individuals free of complications, according to the study question, as efficiently as possible. Cases and controls were unmatched, so that the impact of key variables, such as age, could still be assessed and any adjustments were taken care of in the analyses. The present study includes 477 of these 543 individuals in whom complete data on serum levels of sRAGE, endothelial and renal dysfunction markers, inflammatory markers and other covariates were available. The 66 individuals excluded only differed from the 477 included with regard to BP (higher systolic and diastolic values; data not shown). Levels of sRAGE (as well as AGEs, markers of inflammation and endothelial dysfunction) could not be determined at baseline because the blood samples had run out.

\section{Main determinant}

Plasma levels of sRAGE were measured using a commercially available ELISA kit (Quantikine; R\&D systems, Minneapolis, MN, USA) according to the manufacturer's protocol. Briefly, a monoclonal antibody generated against the N-terminal extracellular domain of human RAGE was used to capture sRAGE from plasma. Captured sRAGE was detected with a polyclonal anti-human sRAGE antibody. After washing, plates were incubated with streptavidin-horseradish peroxidase, developed with appropriate substrate, and $\mathrm{OD}_{450}$ was determined using an ELISA plate reader. Measurements were performed in duplicate and the intra-assay and interassay CV values were $2.9 \%$ and $11.5 \%$, respectively.

\section{Main outcomes}

Macrovascular disease CVD was defined as a positive medical history of a cardiovascular event, including myocardial infarction, angina, coronary artery bypass graft and/or stroke, and/or ischaemic changes on a centrally Minnesota-coded ECG [34].

Microvascular disease Albumin excretion rates (UAEs) were measured from duplicate $24 \mathrm{~h}$ urine collections [27] and micro- and macroalbuminuria were defined as a UAE between 20 and 200, and above $200 \mu \mathrm{g} / \mathrm{min}$, respectively. We also calculated the estimated glomerular filtration rates (eGFRs) for the individuals using the Cockcroft-Gault formula [35]. Retinopathy was assessed from retinal photographs according to the EURODIAB protocol; non-proliferative retinopathy was defined as the presence of one or more microaneurysms, haemorrhages, and/or hard exudates. Proliferative retinopathy was defined as any new vessels, fibrous proliferations, pre-retinal haemorrhage, vitreous haemorrhage or photocoagulation scars [36].

\section{Other risk factors}

BP was recorded twice with a random zero sphygmomanometer (Hawskley, Lancing, UK). Hypertension was defined as systolic pressure $\geq 140 \mathrm{mmHg}$, diastolic pressure $\geq 90 \mathrm{mmHg}$ and/or use of antihypertensive drugs. Smoking habits were ascertained by questionnaire and individuals were categorised into categories: 'non', 'moderate' and 'heavy smokers', according to their levels of pack-years smoked (none, and below or above the sex-specific median, respectively). Weight and height were measured with indoor clothing without shoes and BMI calculated. Blood samples were taken, fasting if possible, for measurement of lipids and glycaemic control. Cholesterol and triacylglycerol levels were measured by enzymatic colorimetric tests [37], and HDL-cholesterol was measured directly [38]. LDL-cholesterol was calculated from Friedewald's formula [39]. $\mathrm{HbA}_{1 \mathrm{c}}$ was measured by a latex-enhanced turbidimetric immunoassay (Roche Products, Welwyn Garden City, UK). The reference range for this assay was $4.2-6.2 \%$.

\section{Potential mediators}

Markers of endothelial dysfunction Soluble vascular cell adhesion molecule-1 (sVCAM-1) and soluble E-selectin (sE-selectin) were measured in duplicate by sandwich enzyme immunoassays (R\&D Systems, Oxford, UK). The mean of the duplicates is presented. The intra- and interassay CV values for sVCAM-1 were 4.0 and $9.1 \%$, respectively, and for sE-selectin, 2.1 and 3.1\%, respectively.

Inflammatory markers Plasma levels of C-reactive protein (CRP) were measured with a highly sensitive in-house ELISA [23] and plasma levels of IL-6 and TNF- $\alpha$ were 
measured using commercially available ELISA kits (R\&D Systems) [23]. Intra- and inter-assay CV values were 3.9\% and $8.7 \%, 4.5 \%$ and $9.0 \%$, and $7.3 \%$ and $8.5 \%$, respectively.

AGEs Pentosidine levels were determined in unhydrolysed urine [40]. Urinary excretion of pentosidine was normalised for urine concentration by expressing it as nmol pentosidine/mmol urinary creatinine. Protein-bound $N^{\varepsilon}$-(carboxymethyl)lysine (CML) and $N^{\varepsilon}$-(carboxyethyl)lysine (CEL) were determined in plasma as previously described [41] with an inter-assay $\mathrm{CV}$ of $6.0 \%$. CML and CEL were normalised for lysine concentration.

\section{Statistical analyses}

All analyses were performed with the Statistical Package for Social Sciences (SPSS) version 15.0 for Windows (SPSS, Chicago, IL, USA). Variables with a skewed distribution (i.e. triacylglycerols, CRP, IL-6, TNF- $\alpha$ and pentosidine) were $\log _{e}$ transformed prior to further analyses. Comparisons of characteristics between individuals with or without vascular complications were performed with Student's $t$ or $\chi^{2}$ tests, as appropriate. Cases were all those individuals with CVD or retinopathy or micro- or macroalbuminuria $(n=299)$; controls were all those who had no evidence of CVD, or retinopathy, and were normoalbuminuric $(n=178)$.

Linear regression analyses were used to examine whether sRAGE levels differed between individuals with or without CVD, and similarly, between those with or without microvascular complications. In the analyses comparing individuals with or without CVD, we excluded those in whom CVD was absent but who did have any of the other vascular complications (183 out of 361 individuals). The same control group $(n=178)$ was used in the analyses comparing individuals with or without albuminuria and individuals with or without retinopathy. These analyses were first adjusted for age, sex, $\mathrm{HbA}_{1 \mathrm{c}}$ and duration of diabetes, and then further adjusted for other cardiovascular risk factors (i.e. BMI, triacylglycerols, HDL- and LDL-cholesterol, systolic BP and smoking). Linear regression analyses were also used to examine the extent to which sRAGE levels were associated with endothelial dysfunction (comprising an averaged $z$ score of sVCAM-1 and sE-selectin), inflammation (comprising an averaged $z$ score of CRP, IL- 6 and TNF- $\alpha$ ) and eGFR. Results of these analyses are expressed in standardised regression coefficients $(95 \%$ CIs) to allow the comparison of the strength of the association between sRAGE and each of these variables/scores. Finally, successive adjustments for these variables were performed in order to investigate the extent to which these variables explained (i.e. attenuated) the differences in sRAGE levels between individuals with and without complications.
Because we measured the markers of the potential mediators only once, the associations observed will tend to be diluted. This is the reason why we combined the markers per mechanism (i.e. inflammation and endothelial dysfunction) to yield an inflammatory and an endothelial dysfunction marker $z$ score. We thereby assumed that each marker contributes equally to the activity of the mechanism to which it belongs.

\section{Results}

Table 1 shows the characteristics of the individuals with vascular complications (cases) compared with those without (controls).

Associations between sRAGE and cardiovascular and microvascular disease

Individuals with CVD had higher levels of sRAGE than those without CVD: $\beta=0.16 \mathrm{ng} / \mathrm{ml}$ (95\% CI $0.05-0.26)$ (Table 2, model 1; Fig. 1). Adjustments for other risk factors did not change this difference (model 2).

sRAGE levels (in $\mathrm{ng} / \mathrm{ml}$ ) were also increased across the levels of severity of albuminuria $(\beta=0.04[-0.08,0.16]$ and $\beta=0.13$ [0.01-0.25]) in individuals with micro- and macroalbuminuria compared with those with normoalbuminuria, respectively ( $p$ for trend $=0.031$ ) and similarly across the levels of severity of retinopathy $(\beta=0.06[-0.04,0.15]$ and $\beta=0.12[0.01-0.22])$ in those with non-proliferative and proliferative compared with those without retinopathy, respectively ( $p$ for trend $=0.037$; Table 2 ; model 1 ; Fig. 1 ). These differences were not markedly attenuated after further adjustments for other risk factors but were no longer statistically significant (model 2).

Association between SRAGE and markers of endothelial and renal dysfunction, low-grade inflammation and AGEs

sRAGE was positively and significantly associated with endothelial dysfunction, low-grade inflammation and AGE scores, and even more strongly so with levels of eGFR (Table 3; model 1). Adjustments for other cardiovascular risk factors did not materially change these associations (model 2).

Mediating effects of endothelial and renal dysfunction and low-grade inflammation in the associations between sRAGE and cardiovascular and microvascular complications

The differences in sRAGE between individuals with CVD compared with those without were attenuated after adjust- 
Table 1 Clinical characteristics of the study population $(n=477)$

\begin{tabular}{|c|c|c|c|}
\hline Characteristic & Vascular complications $(n=299)$ & No vascular complications $(n=178)$ & $p$ value \\
\hline Age (years) & $41.7(10.4)$ & $35.9(8.1)$ & $<0.001$ \\
\hline Sex, male/female $(\%)$ & $54 / 46$ & $46 / 54$ & 0.072 \\
\hline BMI $\left(\mathrm{kg} / \mathrm{m}^{2}\right)$ & $24.9(3.5)$ & $23.7(2.6)$ & $<0.001$ \\
\hline $\mathrm{HbA}_{1 \mathrm{c}}(\%)$ & $9.0(1.6)$ & $7.8(1.3)$ & $<0.001$ \\
\hline Duration of diabetes (years) & $25.2(9.1)$ & $15.3(7.0)$ & $<0.001$ \\
\hline LDL-cholesterol (mmol/l) & $3.25(1.09)$ & $2.87(0.90)$ & $<0.001$ \\
\hline HDL-cholesterol (mmol/l) & $1.59(0.42)$ & $1.69(0.45)$ & 0.022 \\
\hline Triacylglycerol (mmol/l) & $1.14(0.85-1.58)$ & $0.85(0.66-1.09)$ & $<0.001$ \\
\hline Smoking: none/moderate/heavy (\%) & $39 / 27 / 34$ & $51 / 31 / 18$ & $<0.001$ \\
\hline Diastolic BP (mmHg) & $75(12)$ & $73(11)$ & 0.048 \\
\hline Systolic BP (mmHg) & $127(21)$ & $114(14)$ & $<0.001$ \\
\hline Serum creatinine $(\mu \mathrm{mol} / \mathrm{l})$ & $76(68-90)$ & $71(64-78)$ & $<0.001$ \\
\hline eGFR $\left(\mathrm{ml} \mathrm{min}{ }^{-1} 1.73 \mathrm{~m}^{-2}\right)$ & $68(22)$ & $80(17)$ & $<0.001$ \\
\hline CVD $(\%)$ & 38.8 & - & - \\
\hline Albuminuria: normo-/micro-/macro- $(\%)$ & $38.5 / 24.1 / 37.5$ & - & - \\
\hline Retinopathy: none/background/proliferative (\%) & $11.7 / 41.1 / 47.2$ & - & - \\
\hline $\mathrm{CRP}(\mathrm{mg} / \mathrm{l})$ & $1.30(0.46-2.80)$ & $0.71(0.35-1.82)$ & $<0.001$ \\
\hline IL-6 (pg/ml) & $2.13(1.35-3.94)$ & $1.55(1.05-2.49)$ & $<0.001$ \\
\hline $\mathrm{TNF}-\alpha(\mathrm{pg} / \mathrm{ml})$ & $3.17(2.40-4.41)$ & $2.23(1.68-2.85)$ & $<0.001$ \\
\hline sE-selectin (ng/ml) & $36(17)$ & $31(11)$ & $<0.001$ \\
\hline sVCAM-1(ng/ml) & $437(147)$ & $379(104)$ & $<0.001$ \\
\hline $\operatorname{CEL}\left(\mu \mathrm{mol}[\mathrm{mmol} \text { lysine }]^{-1}\right)$ & $0.030(0.012)$ & $0.031(0.011)$ & 0.896 \\
\hline $\mathrm{CML}\left(\mu \mathrm{mol}[\mathrm{mmol} \text { lysine }]^{-1}\right)$ & $0.058(0.020)$ & $0.055(0.015)$ & 0.097 \\
\hline Pentosidine (nmol $[\mathrm{mmol} \text { urinary creatinine }]^{-1}$ ) & $0.46(0.33-0.67)$ & $0.43(0.32-0.56)$ & 0.003 \\
\hline
\end{tabular}

Data are means (SD), median (interquartile range) or percentages, as appropriate

CEL, $N^{\varepsilon}$-(carboxyethyl)lysine; CML, $N^{\varepsilon}$-(carboxymethyl)lysine

ments for markers of endothelial dysfunction and low-grade inflammation and more strongly so after adjustments for eGFR (Table 2, models 3-5 compared with model 2). These variables explained about $13 \%, 20 \%$ and $33 \%$ of the association between sRAGE levels and the prevalence of CVD, respectively. All together, these potential mediators explained about $50 \%$ (change of $\beta$ from 0.15 in model 2 to 0.08 in model 6) of the differences in sRAGE between individuals with CVD compared with those without. Endothelial dysfunction, low-grade inflammation and particularly eGFR also attenuated the differences in sRAGE levels across the levels of severity of albuminuria and retinopathy (Table 2, models 3-6).

\section{Additional analyses}

In the analyses above (Table 2, model 4), we have considered eGFR (a marker of kidney dysfunction) as a potential mediator in the relationship between sRAGE and vascular complications. However, kidney dysfunction could also be a confounder in the association between sRAGE levels and vascular complications, and our analyses cannot distinguish between these possibilities. Finally, eGFR could also be a risk marker of vascular complications. When analysed as such, we observed that individuals with CVD had lower eGFR than those without $(\beta=-4.66 \mathrm{ml} / \mathrm{min}[95 \%$ CI $-8.75,-0.57]$, after adjustments for other cardiovascular risk factors and sRAGE levels). However, the inverse associations reported between sRAGE and eGFR (Table 3, models 1 and 2), were not attenuated when further adjusted for the presence of vascular disease (Table 3, model 3), which argues against eGFR being a risk marker of vascular disease.

We found a significant effect modification by sex in the association between sRAGE and CVD ( $p$ for sex interaction $=0.008)$, indicating that the association between sRAGE and CVD was stronger in men $(\beta=0.25 \mathrm{ng} / \mathrm{ml}$ $[0.08-0.43])$ than in women $(\beta=0.07 \mathrm{ng} / \mathrm{ml}[-0.07,0.20])$. However, in both sexes the direction of the association was the same and the number of cases and controls did not differ markedly between the two sexes: 55:81 and 61:97 in men and women, respectively. In addition, no effect modification by sex was found in any of the other associations investigated, i.e. in the associations between plasma sRAGE and microvascular complications and in the associations between sRAGE and markers of endothelial and renal dysfunction and inflammation.

We have also explored whether the level of glycaemic control influenced the associations reported herein. We found no evidence for effect modification by $\mathrm{HbA}_{1 \mathrm{c}}$ (our measure of glycaemic control) in the associations exam- 
Table 2 Associations between levels of sRAGE and CVD, albuminuria and retinopathy

\begin{tabular}{|c|c|c|c|c|c|c|c|c|c|c|c|c|c|}
\hline \multirow[t]{3}{*}{ Model } & \multicolumn{3}{|c|}{$\mathrm{CVD}^{\mathrm{a}}$} & \multicolumn{5}{|c|}{ Albuminuria ${ }^{\mathrm{b}}$} & \multicolumn{5}{|c|}{ Retinopathy $^{\mathrm{c}}$} \\
\hline & \multirow[t]{2}{*}{$\beta^{\mathrm{d}}$} & \multirow[t]{2}{*}{$95 \% \mathrm{CI}$} & \multirow[t]{2}{*}{$p$ value } & \multicolumn{2}{|c|}{ Microalbuminuria } & \multicolumn{2}{|c|}{ Macroalbuminuria } & \multirow{2}{*}{$\begin{array}{l}p \text { value } \\
\text { for trend }\end{array}$} & \multicolumn{2}{|c|}{ Non-proliferative } & \multicolumn{2}{|c|}{ Proliferative } & \multirow{2}{*}{$\begin{array}{l}p \text { value } \\
\text { for trend }\end{array}$} \\
\hline & & & & $\beta^{\mathrm{e}}$ & $95 \%$ CI & $\beta^{\mathrm{e}}$ & $95 \%$ CI & & $\beta^{\mathrm{f}}$ & $95 \% \mathrm{CI}$ & $\beta^{\text {f }}$ & $95 \%$ CI & \\
\hline 1 & 0.16 & $0.05,0.26$ & 0.005 & 0.04 & $-0.08,0.16$ & 0.13 & $0.01,0.25$ & 0.031 & 0.06 & $-0.04,0.15$ & 0.12 & $0.01,0.22$ & 0.037 \\
\hline 2 & 0.15 & $0.04,0.27$ & 0.007 & 0.04 & $-0.08,0.16$ & 0.11 & $-0.02,0.24$ & 0.087 & 0.07 & $-0.03,0.17$ & 0.11 & $0.00,0.22$ & 0.057 \\
\hline 3 & 0.13 & $0.02,0.24$ & 0.017 & 0.01 & $-0.11,0.13$ & 0.06 & $-0.07,0.18$ & 0.350 & 0.05 & $-0.05,0.14$ & 0.09 & $-0.02,0.20$ & 0.106 \\
\hline 4 & 0.10 & $-0.01,0.20$ & 0.074 & -0.01 & $-0.13,0.10$ & -0.03 & $-0.15,0.10$ & 0.670 & 0.04 & $-0.06,0.13$ & -0.01 & $-0.12,0.10$ & 0.815 \\
\hline 5 & 0.12 & $0.01,0.23$ & 0.033 & 0.00 & $-0.12,0.13$ & 0.06 & $-0.07,0.19$ & 0.351 & 0.03 & $-0.07,0.13$ & 0.06 & $-0.05,0.17$ & 0.249 \\
\hline 6 & 0.08 & $-0.03,0.18$ & 0.147 & -0.04 & $-0.15,0.08$ & -0.06 & $-0.18,0.07$ & 0.381 & 0.01 & $-0.08,0.11$ & -0.02 & $-0.13,0.09$ & 0.684 \\
\hline
\end{tabular}

a Individuals without CVD but with microvascular complications $(n=183)$ were excluded from these analyses

${ }^{\mathrm{b}}$ Individuals with normoalbuminuria but with CVD or retinopathy $(n=115)$ were excluded from these analyses

${ }^{\mathrm{c}}$ Individuals without retinopathy but with CVD or albuminuria $(n=35)$ were excluded from these analyses

${ }^{\mathrm{d}-\mathrm{f}}$ Regression coefficients $(\beta)$ indicate difference in sRAGE levels (in $\left.\mathrm{ng} / \mathrm{ml}\right)$ between: ${ }^{\mathrm{d}}$ individuals with $(n=116)$ vs without CVD $(n=178)$; ${ }^{\mathrm{e}}$ individuals with micro- $(n=72)$ or macroalbuminuria $(n=112)$ vs those with normoalbuminuria (reference group; $\left.n=178\right)$; ${ }^{\mathrm{f}}$ individuals with nonproliferative $(n=123)$ or proliferative retinopathy $(n=141)$ vs those without retinopathy (reference group; $n=178$ )

Model 1, adjusted for age, sex, duration of diabetes and $\mathrm{HbA}_{1 \mathrm{c}}$

Model 2, model 1+adjustments for other cardiovascular risk factors (i.e. BMI, triacylglycerols, HDL- and LDL-cholesterol, smoking and systolic BP)

Model 3, model 2+endothelial dysfunction $z$ score

Model 4, model 2+eGFR

Model 5, model 2+inflammation $z$ score

Model 6, model 2+all variables in models 3-5

ined, indicating that the (positive) associations between sRAGE and macro- and microvascular complications as well as between sRAGE levels and markers of endothelial and renal dysfunction and inflammation were similar for individuals with better or poorer glycaemic control.

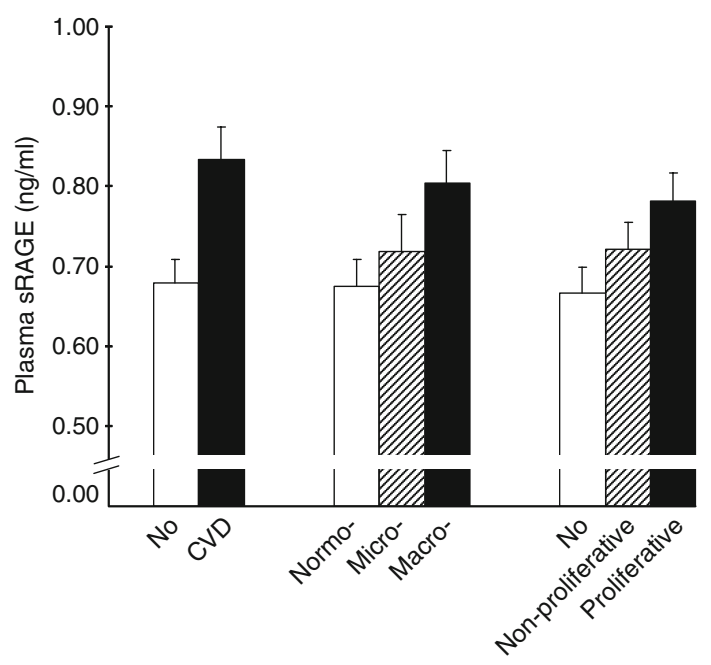

Fig. 1 sRAGE levels according to the presence of CVD and the presence and severity of albuminuria and retinopathy. Bars are means adjusted for age, sex, duration of diabetes and $\mathrm{HbA}_{1 \mathrm{c}}$; error bars indicate SEs of the means. Individuals with CVD $(n=116)$ had higher levels of sRAGE than those without CVD or any microvascular complications $(n=178): p=0.005$. sRAGE levels were higher in the presence and across the levels of severity of albuminuria ( $p$ value for trend $=0.031)$ and retinopathy $(p$ value for trend $=0.037)$

\section{Discussion}

The main finding of this study is that sRAGE levels are positively associated with CVD in type 1 diabetes. This association was independent of other 'traditional' cardiovascular risk factors, but could be partially explained by endothelial and renal dysfunction and low-grade inflammation. Although sRAGE has been investigated in several

Table 3 Associations between sRAGE levels and markers of endothelial and renal dysfunction, low-grade inflammation and AGEs

\begin{tabular}{lcrll}
\hline Dependent variables & Model & $\beta^{\mathrm{a}}$ & \multicolumn{1}{l}{$95 \%$ CI } & $p$ value \\
\hline Endothelial dysfunction & 1 & 0.18 & $0.12,0.24$ & $<0.001$ \\
$z$ score & 2 & 0.17 & $0.11,0.23$ & $<0.001$ \\
eGFR & 1 & -0.30 & $-0.38,-0.23$ & $<0.001$ \\
& 2 & -0.26 & $-0.33,-0.19$ & $<0.001$ \\
& 3 & -0.25 & $-0.32,-0.18$ & $<0.001$ \\
Inflammation $z$ score & 1 & 0.15 & $0.09,0.21$ & $<0.001$ \\
& 2 & 0.14 & $0.09,0.20$ & $<0.001$ \\
AGE $z$ score & 1 & 0.21 & $0.15,0.27$ & $<0.001$ \\
& 2 & 0.20 & $0.14,0.25$ & $<0.001$ \\
\hline
\end{tabular}

${ }^{\mathrm{a}} \beta$, standardised regression coefficient: indicates increase (in SDs) of the dependent variable per $1 \mathrm{SD}$ increase in SRAGE levels

Model 1, adjusted for age, sex, duration of diabetes and $\mathrm{HbA}_{1 \mathrm{c}}$

Model 2, model $1+$ adjustments for other cardiovascular risk factors (i.e. BMI, triacylglycerol, HDL- and LDL-cholesterol, smoking and systolic BP

Model 3, model $2+$ adjustment for the presence of vascular complications 
studies, findings have been scattered. This is the first study that has investigated the associations between sRAGE and macro- and microvascular complications in a large sample of individuals with type 1 diabetes, and has also addressed potential mechanisms that could explain the observed associations.

Human endothelial cells express at least three RAGE variants [4]. The first one is the full-length RAGE, the second, the N-terminally truncated variant with a still unknown function, and the third, the C-terminally truncated soluble form. This naturally occurring form of sRAGE, as well as artificially produced sRAGE, can potentially bind to an AGE ligand thereby acting as a decoy, preventing AGERAGE interaction and activation [4]. This could thus explain any inverse association observed between sRAGE and vascular complications. However, although sRAGE has been an interesting subject of investigation under this suspected decoy function, it is very unlikely that sRAGE can act as such (i.e. by capturing and eliminating AGEs) because the sRAGE concentrations found in plasma are approximately 1,000 times lower than needed for efficient binding and elimination of AGEs [14, 16]. The increased levels of sRAGE in individuals with CVD and across levels of severity of albuminuria and retinopathy, as well as the positive association between sRAGE and AGEs reported herein thus seem not to sustain such a decoy function.

The observed positive association between SRAGE and CVD is in agreement with earlier observations in type 2 diabetes [15] and heart failure patients [42], although opposite findings have been reported in non-diabetic men [43]. In addition, esRAGE and sRAGE levels were inversely associated with carotid IMT $[18,20]$ and its progression [21] in individuals with type 1 diabetes; because carotid IMT is considered as a valid marker of atherosclerosis, which is a strong underlying cause of CVD, these findings seem to contradict the ones reported herein. However, because associations with clinical cardiovascular events were not reported in those studies and we have not measured carotid IMT, we cannot fully judge these apparent discrepancies. Interestingly, in another study in type 2 diabetes both esRAGE and sRAGE were positively, not inversely, associated with carotid IMT, although not significantly so [16].

sRAGE levels were also positively associated with microvascular complications, although these were not independent of other risk factors in the present study. Positive associations between sRAGE levels and albuminuria have been consistently reported previously $[13,16$, 44]. It is important to note that the only study where associations between both esRAGE and sRAGE on the one hand and UAE on the other were investigated in the same population (type 2 diabetes), both soluble forms of RAGE were positively associated with UAE, but sRAGE more strongly (and significantly so) than esRAGE [16]. The increased levels of sRAGE across levels of severity of retinopathy observed in our study were also in line with a previous study [17]. In contrast, individuals with diabetic retinopathy were characterised by lower esRAGE levels compared with individuals without retinopathy [19].

The reasons for the contradictory findings reported above are not clear but may be explained by differences in the study population (i.e. diabetes vs no diabetes) and/or by the fact that different variants of sRAGE are detected by different assays used in different studies, namely the total pool of sRAGE by the Quantikine sRAGE ELISA kit (R\&D Systems, Minneapolis, USA) [13-17] or specifically esRAGE by the B-Bridge International esRAGE ELISA kit (Daiichi Fine Chemicals, Takaoka, Japan) $[16,18,19]$. This is also supported by the increased sRAGE $[13,15,17,20]$, but decreased esRAGE levels [18-20] reported in individuals with diabetes compared with controls. These two assays measure different pools of sRAGE and different variants of sRAGE may have different functions and therefore they are not inter-exchangeable. Indeed, the regulatory mechanisms for proteolytic shedding of cellbound RAGE to generate sRAGE and for alternative splicing to generate esRAGE are still unclear. Recently, new splice variants have been discovered [6] and further studies are necessary to establish which variants of sRAGE are measured by these assays and whether different soluble forms of RAGE have different (patho) physiological functions.

We, as others, have found positive associations between sRAGE and markers of endothelial dysfunction and lowgrade inflammation $[14,45]$. The current view is that the activation of RAGE, a $55 \mathrm{kDa}$ protein and member of the immunoglobulin superfamily of cell-surface receptors, through ligand binding activates the endothelial cell and triggers multiple signalling cascades $[8,9]$. This results in activation and translocation of nuclear transcription factors and transcription of the target genes, including those for VCAM-1 [9], E-selectin [10] and proinflammatory cytokines [11]. AGEs are positive regulators of the cellular RAGE expression [46], and an increased formation of AGEs enhanced the expression and secretion of the soluble forms of RAGE [47, 48]. In addition, the positive association between sRAGE and AGEs observed in this and other studies $[13,14]$ supports the view that sRAGE could be a reflection of RAGE, and that activation of the AGE-RAGE axis can lead to increased endothelial dysfunction and low-grade inflammation, and subsequently to an increased risk of cardiovascular complications in type 1 diabetes.

We have shown that sRAGE is positively and independently associated with renal dysfunction, defined by lower eGFR. This is in line with a recent study in which sRAGE 
(and also esRAGE) were inversely associated with current and follow-up levels of eGFR in a large population of elderly women [49]. In addition, it has been shown that the activation of the AGE-RAGE axis could lead to glomerulosclerosis and glomerular hyperpermeability through the upregulation of vascular endothelial growth factor and/or transforming growth factor- $\beta$ [12]. We have also shown that renal dysfunction mediated the associations between sRAGE levels on the one hand and CVD or microvascular complications on the other. Although there is some evidence to support the assumption of renal dysfunction being a mediator in the causal pathway between sRAGE levels (as a reflection of the activity of the AGE-RAGE axis) and vascular complications, we cannot neglect the possibility of renal dysfunction being a confounder in these associations.

It is unclear why the relationship between sRAGE levels and CVD observed in the present study was stronger in men than in women. sRAGE levels did not differ between the two sexes and no significant effect modification by sex was found in any of the other associations investigated. Therefore, we cannot discard the possibility that this finding was due to chance. In addition, differences (if any) between sexes in the association between sRAGE and vascular complications have not been reported before. Interestingly, women with polycystic ovary syndrome were found to have higher levels of serum AGEs and RAGE expression on circulating monocytes than healthy controls, and these differences may be partly explained by their elevated levels of testosterone [50]. Altogether, these findings suggest that the role of sex hormones in the associations between plasma sRAGE and CVD may need to be further explored.

There are limitations to our study. First, we cannot establish whether the associations are causal due to the cross-sectional design of the study. Further follow-up data on these or other patient groups would, of course, be invaluable, in testing whether our findings are probably due to causal relationships. However, no longitudinal data of this type exist in large groups of individuals with type 1 diabetes at the time, and therefore this cross-sectional study may serve as a reasonable starting point to further explore these associations. Second, our findings are limited to type 1 diabetic individuals and extrapolations to non-diabetic individuals may need caution. Third, although we minimised the influence of confounding in the associations observed by adjustments for many possible confounders, interference by factors we did not measure cannot be ruled out. Fourth, the levels of the mediators investigated were only measured once, which might have diluted the associations we found. However, we combined different markers of the potential mediators in $z$ scores to yield more robust scores. Finally, there may be some concerns with regard to the effects of storage on plasma sRAGE levels. Such effects, if any (e.g. autoglycosylation) are unknown.
However, because the storing time and conditions $\left(-80^{\circ} \mathrm{C}\right)$ were similar to all samples, any bias that may have occurred is likely to have been non-differential and therefore did not affect our overall findings.

In conclusion, in this study of individuals with type 1 diabetes, sRAGE was found to be significantly associated with CVD and this was independent of other cardiovascular risk factors. Endothelial and renal dysfunction and lowgrade inflammation explained about $50 \%$ of this association, suggesting that sRAGE levels as a reflection of RAGE lead to greater prevalence of CVD through increased endothelial and renal dysfunction and low-grade inflammation. Nevertheless, the precise role of sRAGE in the pathophysiology of diabetes and vascular complications needs to be explored further in prospective studies.

Acknowledgements We would like to thank all the investigators and participants who very kindly participated in the study. The EURODIAB Prospective Complications Study was supported by grants from the Wellcome Trust, the European Community and Diabetes UK. I. Ferreira is supported by a postdoctoral research grant from the Netherlands Heart Foundation (NHS; Grant No. 2006T050). Members of the EURODIAB Prospective Complications Study Group are listed in the Electronic supplementary material.

Duality of interest The authors declare that there is no duality of interest associated with this manuscript.

Open Access This article is distributed under the terms of the Creative Commons Attribution Noncommercial License which permits any noncommercial use, distribution, and reproduction in any medium, provided the original author(s) and source are credited.

\section{References}

1. Soedamah-Muthu SS, Fuller JH, Mulnier HE, Raleigh VS, Lawrenson RA, Colhoun HM (2006) High risk of cardiovascular disease in patients with type 1 diabetes in the U.K.: a cohort study using the general practice research database. Diabetes Care 29:798-804

2. Laing SP, Swerdlow AJ, Slater SD et al (2003) Mortality from heart disease in a cohort of 23,000 patients with insulin-treated diabetes. Diabetologia 46:760-765

3. Brownlee M (2001) Biochemistry and molecular cell biology of diabetic complications. Nature 414:813-820

4. Yonekura H, Yamamoto Y, Sakurai S et al (2003) Novel splice variants of the receptor for advanced glycation end-products expressed in human vascular endothelial cells and pericytes, and their putative roles in diabetes-induced vascular injury. Biochem J 370:1097-1109

5. Schlueter C, Hauke S, Flohr AM, Rogalla P, Bullerdiek J (2003) Tissue-specific expression patterns of the RAGE receptor and its soluble forms - a result of regulated alternative splicing? Biochim Biophys Acta 1630:1-6

6. Hudson BI, Carter AM, Harja E et al (2008) Identification, classification, and expression of RAGE gene splice variants. FASEB J 22:1572-1580 
7. Raucci A, Cugusi S, Antonelli A et al (2008) A soluble form of the receptor for advanced glycation endproducts (RAGE) is produced by proteolytic cleavage of the membrane-bound form by the sheddase a disintegrin and metalloprotease 10 (ADAM10). FASEB J 22:3716-3727

8. Kislinger T, Fu C, Huber B et al (1999) $N$ (epsilon)-(carboxymethyl) lysine adducts of proteins are ligands for receptor for advanced glycation end products that activate cell signaling pathways and modulate gene expression. J Biol Chem 274:31740-31749

9. Harja E, Bu DX, Hudson BI et al (2008) Vascular and inflammatory stresses mediate atherosclerosis via RAGE and its ligands in apoE $\mathrm{E}^{-/-}$mice. J Clin Invest 118:183-194

10. Basta G, Lazzerini G, Massaro M et al (2002) Advanced glycation end products activate endothelium through signal-transduction receptor RAGE: a mechanism for amplification of inflammatory responses. Circulation 105:816-822

11. Hofmann MA, Drury S, Fu C et al (1999) RAGE mediates a novel proinflammatory axis: a central cell surface receptor for S100/ calgranulin polypeptides. Cell 97:889-901

12. Wendt TM, Tanji N, Guo J et al (2003) RAGE drives the development of glomerulosclerosis and implicates podocyte activation in the pathogenesis of diabetic nephropathy. Am J Pathol 162:1123-1137

13. Tan KC, Shiu SW, Chow WS, Leng L, Bucala R, Betteridge DJ (2006) Association between serum levels of soluble receptor for advanced glycation end products and circulating advanced glycation end products in type 2 diabetes. Diabetologia 49:2756-2762

14. Nakamura K, Yamagishi S, Adachi H et al (2008) Serum levels of soluble form of receptor for advanced glycation end products (sRAGE) are positively associated with circulating AGEs and soluble form of VCAM-1 in patients with type 2 diabetes. Microvasc Res 76:52-56

15. Nakamura K, Yamagishi S, Adachi H et al (2007) Elevation of soluble form of receptor for advanced glycation end products (sRAGE) in diabetic subjects with coronary artery disease. Diabetes Metab Res Rev 23:368-371

16. Humpert PM, Djuric Z, Kopf S et al (2007) Soluble RAGE but not endogenous secretory RAGE is associated with albuminuria in patients with type 2 diabetes. Cardiovasc Diabetol 6:9

17. Challier M, Jacqueminet S, Benabdesselam O, Grimaldi A, Beaudeux JL (2005) Increased serum concentrations of soluble receptor for advanced glycation endproducts in patients with type 1 diabetes. Clin Chem 51:1749-1750

18. Koyama H, Shoji T, Yokoyama H et al (2005) Plasma level of endogenous secretory RAGE is associated with components of the metabolic syndrome and atherosclerosis. Arterioscler Thromb Vasc Biol 25:2587-2593

19. Katakami N, Matsuhisa M, Kaneto H et al (2005) Decreased endogenous secretory advanced glycation end product receptor in type 1 diabetic patients: its possible association with diabetic vascular complications. Diabetes Care 28:2716-2721

20. Katakami N, Matsuhisa M, Kaneto H et al (2008) Endogenous secretory RAGE but not soluble RAGE is associated with carotid atherosclerosis in type 1 diabetes patients. Diab Vasc Dis Res 5:190-197

21. Katakami N, Matsuhisa M, Kaneto H et al (2009) Serum endogenous secretory RAGE level is an independent risk factor for the progression of carotid atherosclerosis in type 1 diabetes. Atherosclerosis doi:101016/jatherosclerosis200808026

22. Schram MT, Chaturvedi N, Schalkwijk CG, Fuller JH, Stehouwer CD (2005) Markers of inflammation are cross-sectionally associated with microvascular complications and cardiovascular disease in type 1 diabetes - the EURODIAB Prospective Complications Study. Diabetologia 48:370-378

23. Schram MT, Chaturvedi N, Schalkwijk C et al (2003) Vascular risk factors and markers of endothelial function as determinants of inflammatory markers in type 1 diabetes: the EURODIAB Prospective Complications Study. Diabetes Care 26:2165-2173

24. Schiffrin EL, Lipman ML, Mann JF (2007) Chronic kidney disease: effects on the cardiovascular system. Circulation 116:85-97

25. Stephenson J, Fuller J (1994) Microvascular and acute complications in IDDM patients: the EURODIAB IDDM Complications Study. Diabetologia 37:278-285

26. Chaturvedi N, Sjoelie AK, Porta M et al (2001) Markers of insulin resistance are strong risk factors for retinopathy incidence in type 1 diabetes. Diabetes Care 24:284-289

27. Chaturvedi N, Bandinelli S, Mangili R, Penno G, Rottiers RE, Fuller JH (2001) Microalbuminuria in type 1 diabetes: rates, risk factors and glycemic threshold. Kidney Int 60:219-227

28. Soedamah-Muthu SS, Colhoun HM, Abrahamian H et al (2002) Trends in hypertension management in type I diabetes across Europe, 1989/1990-1997/1999. Diabetologia 45:1362-1371

29. Schram MT, Schalkwijk CG, Bootsma AH, Fuller JH, Chaturvedi N, Stehouwer CD (2005) Advanced glycation end products are associated with pulse pressure in type 1 diabetes: the EURODIAB Prospective Complications Study. Hypertension 46:232-237

30. Chaturvedi N, Schalkwijk CG, Abrahamian H, Fuller JH, Stehouwer CD (2002) Circulating and urinary transforming growth factor beta1, Amadori albumin, and complications of type 1 diabetes: the EURODIAB prospective complications study. Diabetes Care 25:2320-2327

31. Soedamah-Muthu SS, Chaturvedi N, Teerlink T, Idzior-Walus B, Fuller JH, Stehouwer CD (2005) Plasma homocysteine and microvascular and macrovascular complications in type 1 diabetes: a cross-sectional nested case-control study. J Intern Med 258:450-459

32. Schalkwijk CG, Chaturvedi N, Schram MT, Fuller JH, Stehouwer CD (2006) Adiponectin is inversely associated with renal function in type 1 diabetic patients. J Clin Endocrinol Metab 91:129-135

33. Gruden G, Bruno G, Chaturvedi N et al (2008) Serum heat shock protein 27 and diabetes complications in the EURODIAB prospective complications study: a novel circulating marker for diabetic neuropathy. Diabetes 57:1966-1970

34. Koivisto VA, Stevens LK, Mattock M et al (1996) Cardiovascular disease and its risk factors in IDDM in Europe. EURODIAB IDDM Complications Study Group. Diabetes Care 19:689-697

35. Cockcroft DW, Gault MH (1976) Prediction of creatinine clearance from serum creatinine. Nephron 16:31-41

36. Aldington SJ, Kohner EM, Meuer S, Klein R, Sjolie AK (1995) Methodology for retinal photography and assessment of diabetic retinopathy: the EURODIAB IDDM complications study. Diabetologia 38:437-444

37. Fossati P, Prencipe L (1982) Serum triglycerides determined colorimetrically with an enzyme that produces hydrogen peroxide. Clin Chem 28:2077-2080

38. Sugiuchi H, Uji Y, Okabe H et al (1995) Direct measurement of high-density lipoprotein cholesterol in serum with polyethylene glycol-modified enzymes and sulfated alpha-cyclodextrin. Clin Chem 41:717-723

39. Friedewald WT, Levy RI, Fredrickson DS (1972) Estimation of the concentration of low-density lipoprotein cholesterol in plasma, without use of the preparative ultracentrifuge. Clin Chem 18:499-502

40. Smulders RA, Stehouwer CD, Schalkwijk CG, Donker AJ, van Hinsbergh VW, TeKoppele JM (1998) Distinct associations of $\mathrm{HbAlc}$ and the urinary excretion of pentosidine, an advanced glycosylation end-product, with markers of endothelial function in insulin-dependent diabetes mellitus. Thromb Haemost 80:52-57

41. Lieuw-A-Fa ML, van Hinsbergh VW, Teerlink T et al (2004) Increased levels of $N$ (epsilon)-(carboxymethyl)lysine and $N($ epsilon)-(carboxyethyl)lysine in type 1 diabetic patients with impaired renal function: correlation with markers of endothelial dysfunction. Nephrol Dial Transplant 19:631-636 
42. Koyama Y, Takeishi Y, Niizeki T et al (2008) Soluble receptor for advanced glycation end products (RAGE) is a prognostic factor for heart failure. J Card Fail 14:133-139

43. Falcone C, Emanuele E, D'Angelo A et al (2005) Plasma levels of soluble receptor for advanced glycation end products and coronary artery disease in nondiabetic men. Arterioscler Thromb Vasc Biol 25:1032-1037

44. Humpert PM, Kopf S, Djuric Z et al (2006) Plasma sRAGE is independently associated with urinary albumin excretion in type 2 diabetes. Diabetes Care 29:1111-1113

45. Nakamura K, Yamagishi S, Adachi H et al (2007) Serum levels of sRAGE, the soluble form of receptor for advanced glycation end products, are associated with inflammatory markers in patients with type 2 diabetes. Mol Med 13:185-189

46. Tanaka N, Yonekura H, Yamagishi S, Fujimori H, Yamamoto Y, Yamamoto H (2000) The receptor for advanced glycation end products is induced by the glycation products themselves and tumor necrosis factor-alpha through nuclear factor-kappa B, and by 17 beta-estradiol through $\mathrm{Sp}-1$ in human vascular endothelial cells. J Biol Chem 275:25781-25790

47. Miura J, Yamamoto Y, Osawa M et al (2007) Endogenous secretory receptor for advanced glycation endproducts levels are correlated with serum pentosidine and CML in patients with type 1 diabetes. Arterioscler Thromb Vasc Biol 27:253-254

48. Yamagishi S, Adachi H, Nakamura K et al (2006) Positive association between serum levels of advanced glycation end products and the soluble form of receptor for advanced glycation end products in nondiabetic subjects. Metabolism 55:1227-1231

49. Semba RD, Ferrucci L, Fink JC et al (2009) Advanced glycation end products and their circulating receptors and level of kidney function in older community-dwelling women. Am J Kidney Dis 53:51-58

50. Diamanti-Kandarakis E, Piperi C, Kalofoutis A, Creatsas G (2005) Increased levels of serum advanced glycation end-products in women with polycystic ovary syndrome. Clin Endocrinol (Oxf) $62: 37-43$ 